\title{
Urgent surgical treatment for a huge metastatic tumor of the right lung via plastron thoracotomy: A case report
}

\author{
Ryuta Fukai ${ }^{1}$, Yoshihito Irie ${ }^{2}$, Yoshiaki Katada ${ }^{3}$, and Hajime Arifuku ${ }^{4}$ \\ ${ }^{1}$ Department of General Thoracic Surgery, Shonan Kamakura General Hospital, Japan \\ ${ }^{2}$ Department of Cardiovascular Surgery, Iwaki Kyoritus General Hospital, Japan \\ ${ }^{3}$ Department of Radiology, Tokyo Medical and Dental University, Japan \\ ${ }^{4}$ Department of Respiratory Medicine, Dokkyo University Koshigya Hospital, Japan
}

\begin{abstract}
For an extremely large intrathoracic tumor, which almost occupies a unilateral thoracic cavity, it is likely that salvage surgery would provide benefit. Nevertheless, the operative risk of such salvage surgery is very high, plus the surgery itself often becomes challenging. The choice of surgical approach presents another problem, as the presence of a large tumor mass often makes it difficult to secure a workable surgical field. Our patient was a 63-year old man with a huge right lung metastasis from a left crural myxofibrosarcoma. We performed an urgent salvage surgery: a right pneumonectomy with assisted circulation via plastron thoracotomy. The patient lived a year and two months after the operation. We believe that plastron thoracotomy can be one of the available choices for surgical approach for resection of a giant thoracic tumor.
\end{abstract}

\section{Introduction}

We rarely encounter patients with tumors so giant that they require urgent, life-saving operations. Such surgeries carry high perioperative risk and are often technically challenging, therefore we tend to decline performing such surgeries. However, there are cases where an operation is truly needed to preserve life. Furthermore, surgical approach is vitally important: there is considerable difficulty in ensuring an adequate surgical field due to the magnitude of the tumor, which even affect cardiac hemodynamics. In fact, in the case of such an enormous thoracic tumor, we are not able to use the lateral position, as this would compress the heart itself. For the present case, we experienced a huge right pulmonary metastasis from a left cnemial myxofibrosarcoma, and we successfully performed a right pneumonectomy with assisted circulation via plastron thoracotomy (PT).

\section{Case report}

A 63-year-old man visited our hospital complaining of general malaise, reduced appetite, and weight loss. On chest $\mathrm{x}$-ray, there was an infiltrative shadow from the right middle to lower lung fields, and a very large mass shadow was recognized in the retrosternal space on the lateral view (Figure 1).

Computed tomography (CT) showed a huge right intrathoracic tumor with pleural effusion, which occupied nearly the entire right thoracic space (Figure 2). Urgent hospital admission was recommended. Following CT-guided biopsy, it was suspected that the present tumor was a pulmonary metastasis of the myxofibrosarcoma. The patient had undergone resection of a left crural myxofibrosarcoma five years ago at another hospital, and concurrently had been detected a small pulmonary nodule in the right lung. However, he had stopped going to hospital arbitrarily. Initial laboratory findings showed anemia $(\mathrm{Hb}$ $10.2 \mathrm{~g} / \mathrm{dl}$ ), hypoproteinemia (TP $6.1 \mathrm{~g} / \mathrm{dl}$, Alb $2.47 \mathrm{~g} / \mathrm{dl}$ ), and elevated C-reactive protein $(18.28 \mathrm{mg} / \mathrm{dl})$. No other lesions were detected in the patient's body following thorough systemic examination. Cardiac function was preserved, although mild hypoxia was detected (Table 1).

We recommended urgent, life-saving tumor resection, because constitutional symptoms, such as malaise and weight loss, appeared to be due to this giant tumor. We performed transcatheter arterial embolization (TAE) of the bronchial arteries of the right middle and lower lobe, the fourth through the ninth right intercostal arteries, the right inferior phrenic artery, and the right internal mammary artery

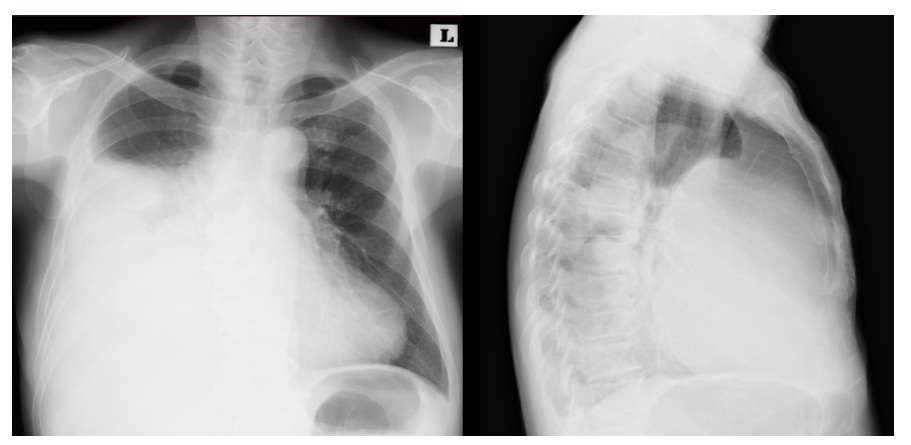

Figure 1. Chest X-rays show a very large shadow from the right middle to lower lung fields at the retrosternal space.

Correspondence to: Ryuta Fukai, Department of General Thoracic Surgery, Shonan Kamakura General Hospital, 1370-1, Okamoto, Kamakura, Kanagawa, 247-8533, Japan, Tel: +81 46746 1717; Fax: +81 46747 8243; E-mail: ryuta.f@ hotmail.co.jp

Key words: plastron thoracotomy, giant thoracic tumor, life-saving operation, assisted circulation

Received: February 22, 2017; Accepted: March 24, 2017; Published: March 27, 2017 


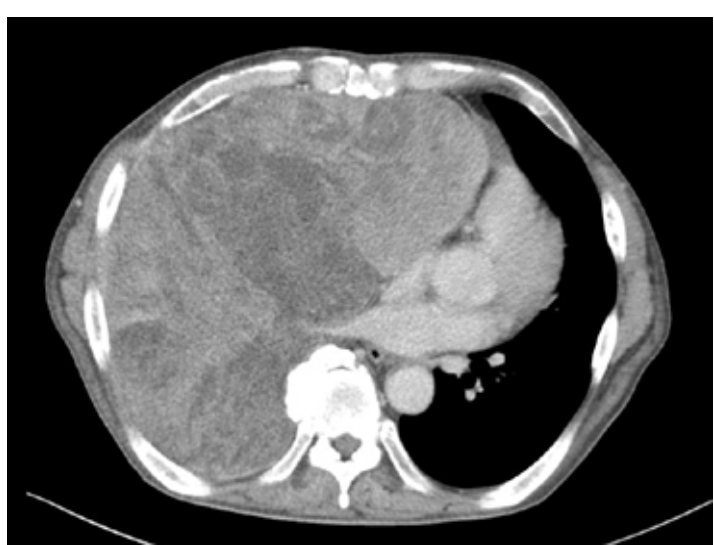

Figure 2. CT shows a huge mass, which occupies nearly the whole right thoracic cavity, with a small amount of pleural effusion.

Table 1. Laboratory findings on admission.

\begin{tabular}{|c|c|c|c|}
\hline WBC & $8700 / \mu 1$ & $\mathrm{TP}$ & $6.1 \mathrm{~g} / \mathrm{dl}$ \\
\hline $\mathrm{Hb}$ & $10.2 \mathrm{~g} / \mathrm{dl}$ & $\mathrm{Alb}$ & $2.47 \mathrm{~g} / \mathrm{dl}$ \\
\hline $\mathrm{Plt}$ & $32.9 \times 10^{4} / \mu \mathrm{l}$ & $\mathrm{CRP}$ & $18.28 \mathrm{mg} / \mathrm{dl}$ \\
\hline $\mathrm{GOT}$ & $26 \mathrm{U} / \mathrm{L}$ & $\mathrm{PaO}_{2}$ & $76.4 \mathrm{mmHg}$ \\
\hline $\mathrm{GPT}$ & $24 \mathrm{U} / \mathrm{L}$ & $\mathrm{PaCO}_{2}$ & $41.1 \mathrm{mmHg}$ \\
\hline $\mathrm{LDH}$ & $129 \mathrm{U} / \mathrm{L}$ & $(\mathrm{O} 2$ mask $3 \mathrm{~L} / \mathrm{min})$. & \\
\hline $\mathrm{T}-\mathrm{Bil}$ & $1.12 \mathrm{mg} / \mathrm{dl}$ & & \\
\hline $\mathrm{BUN}$ & $17 \mathrm{mg} / \mathrm{dl}$ & $\mathrm{VC}$ & $1.79 \mathrm{~L}(52.8 \%)$ \\
\hline $\mathrm{Cr}$ & $0.9 \mathrm{mg} / \mathrm{dl}$ & $\mathrm{FEV}$ & 1.0 \\
\hline $\mathrm{Na}$ & $138 \mathrm{mmol} / 1$ & UCG: & \\
\hline $\mathrm{K}$ & $5.2 \mathrm{mmol} / 1$ & ejection fraction & $62 \% .66 \%)$ \\
\hline $\mathrm{Cl}$ & $102 \mathrm{mmol} / 1$ & cardiac index & $3.4 \mathrm{~L} / \mathrm{min} / \mathrm{m}^{2}$ \\
\hline
\end{tabular}

the day before the operation for prophylactic control of intraoperative bleeding.

We had planned a clamshell incision (CI) at first, but we chose PT after taking into consideration the following points. First, we believed PT would be less burdensome than CI, because we considered the use of assisted circulation for securing a view of the right hilum; we decided not to use epidural anesthesia. Second, PT would be less possible to leads to contralateral dissemination, which arise from expanding surgical field to the left thoracic cavity.

We made our incision at the midline of the sternum, dissected subcutaneous tissue away from the anterior chest wall, and ligated and sundered the internal thoracic artery and vein at the level of the second intercostal space. We cut from the third to fifth front ribs bilaterally, and transected the sternum at the level of the second intercostal space. We cut each intercostal muscle, crushed the sixth and seventh ribs at the costal arch, and finally reversed the sternum with transected ribs caudally (Figure 3). We located the huge mass in the center of the surgical field and barely recognized the heart beat at the left inferior corner of the field. We dissected the tumor from the pericardium as much as possible; however, we could not appreciate the right pulmonary hilum in the right thoracic cavity and consequently opened the pericardium. We were able to obtain a view of the right hilum using assisted circulation because of the pressure decrease, which was assisted by manual cardiac compression. We transected the right main pulmonary artery between the superior vena cava and the ascending aorta, pulmonary veins in the pericardium, and the right main bronchus just distal to the stump of the pulmonary artery. Thereafter, the tumor and the right lung were removed from the right thoracic cavity after exfoliation from the posterior chest wall and diaphragm. We were able to avoid opening the left thoracic cavity due to careful dissection. After repeated irrigation of the surgical field, the transected sternum and each rib were fixed with wires.

In the resected specimen, the right upper lobe was occupied by tumor tissue with degeneration and necrosis, and the middle and lower lobes were collapsed markedly.

Microscopic findings showed atypical spindle cells, which were identified as mesenchymal and were vimentin-positive. Sixty percent of the sample was positive for anti-Ki67 antibody. We diagnosed the mass as pulmonary metastasis of myxofibrosarcoma (Figure 4).

The patient was discontinued from mechanical ventilation on the forth postoperative day. He needed paracentesis of pleural effusion three times; however, there was no major wound complication (Figure 5 ). The patient was discharged on the $38^{\text {th }}$ postoperative day. He lived a year and two months after the operation owing to effective adjuvant radiation, although he had additional lung and liver metastasis.

\section{Discussion}

Sometimes an urgent salvage operation is required, due to systemic breakdown and weight loss in a patient with a huge tumor which nearly occupies the entire unilateral thoracic space. In this case, we conducted a life-saving right pneumonectomy for pulmonary metastasis of myxofibrosarcoma that originated from the left lower thigh via plastron thoracotomy with assisted circulation. The patient survived 1 year and 2 months. While we might naturally hesitate whether or not to perform surgery on a very large mass, it seems worthwhile to consider aggressive operation in combination with multimodal therapy if there is a chance to improve prognosis.

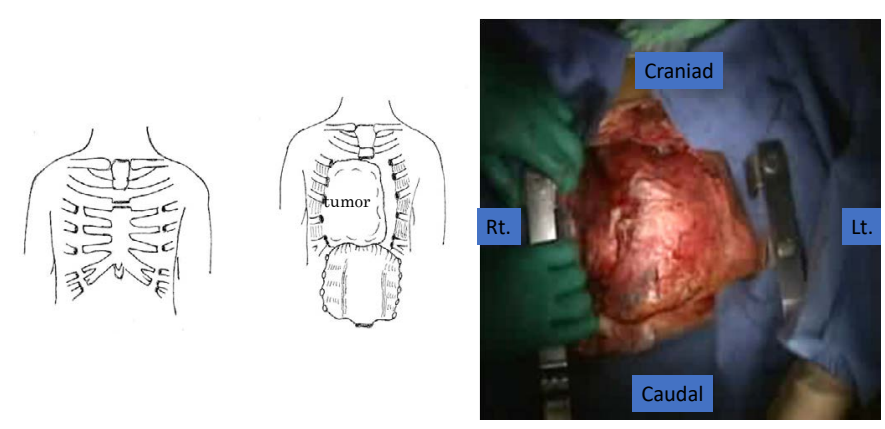

Figure 3. The resected anterior chest wall, which is continuous with caudal structures such as skin, subcutaneous tissue, and rectus abdominis, was turned caudally.

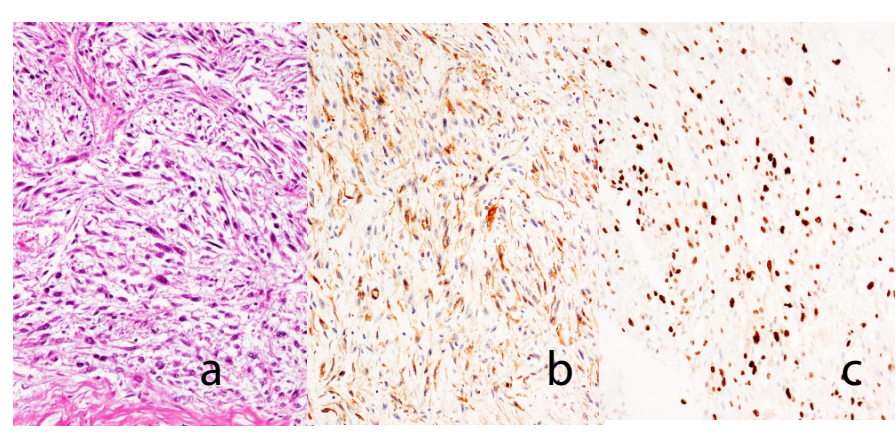

Figure 4. Microscopic findings of the resected specimen.

a) Hematoxylin and eosin stain $(\times 200)$.

b) Tumor cell demonstrating immunopositivity for vimentin $(\times 200)$.

c) Tumor cell demonstrating more than sixty percent immunopositivitiy for anti-Ki67 antibody $(\times 200)$. 
Various approaches to the chest have been noted: posterolateral, median sternotomy, and $\mathrm{CI}$, the choice of which incision to perform depends on the tumor site and involved structures [1]. Lateral recumbent position is essentially impossible because of mass effect on the heart when resecting a huge intrathoracic tumor. Many articles have reported the benefits of CI, which provides sufficient exposure of the mediastinum, the involved hemi-thorax, the pulmonary hilum, and anterior cervicothoracic junction [1]. It also gives better access to the lower lobe than median sternotomy [2]. PT, which has been reported in the resection of a giant invasive thymic tumor, consists of a T-shape incision on the precordium, transection of the sternum at the first intercostal space, severing of the second to the seventh bilateral costal cartilages anteriorly, and inversion of the anterior chest wall [3]. PT has several advantages compared with CI (Table 2). We chose PT because it is less painful and the skin incision is simpler. Moreover, PT does not require bilateral thoracotomy, which is very important for avoiding contralateral dissemination.

In our case we obtained our surgical field by transecting the sternum at the second intercostal space in consideration of our tumor margin, and we cut from the third to the seventh bilateral costal cartilages, thus

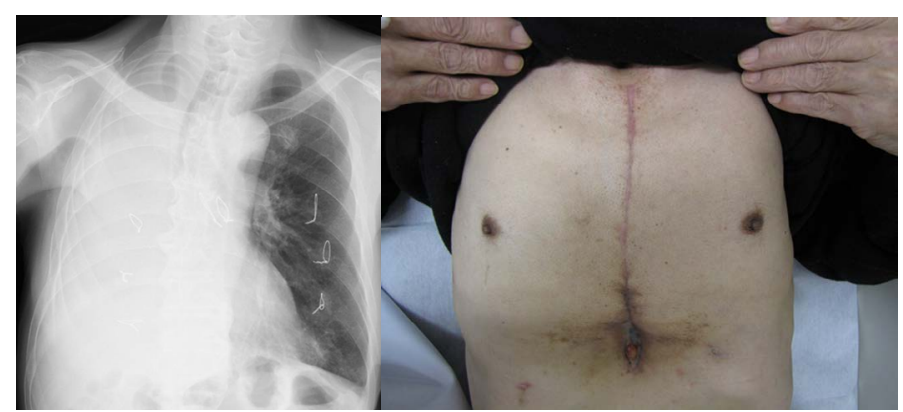

Figure 5.Postoperative chest X-ray and a picture of the incision site (one month after the operation).

Table 2. Comparison between plastron thoracotomy and clamshell incision.

\begin{tabular}{|c|c|c|}
\hline & Plastron thoracotomy & Clamshell incision \\
\hline Incision & Longitudinal & Horizontal \\
\hline Bone transection & Sternum, Ribs & Sternum \\
\hline Pain & Mild & Moderate or Severe \\
\hline Vascular transection & $\begin{array}{c}\text { Internal arteries \& Intercostal } \\
\text { arteries }\end{array}$ & Internal arteries \\
\hline Thoracotomy & Unilateral & Bilateral \\
\hline
\end{tabular}

the operative field was adequate for conducting right pneumonectomy with assisted circulation with only right thoracotomy. It seems that PT results in more wound-healing difficulties compared with CI because it sacrifices more intercostal arteries, however, our case had no major wound complications. It should be borne in mind, however, that although PT was chosen in our case, it is not that we assert PT should be the first choice in most cases of giant intrathoracic tumor. Further studies should be necessary to validate our choice as we usually experience a limited number of such cases.

We could not expose the right pulmonary hilum in the right thorax because of the huge mass, therefore, we opened the pericardium. Nevertheless, the patient had a decrease in blood pressure due to compression of the heart when dissecting the tumor. We conducted assisted circulation, thus hemodynamics became adequate for the right pneumonectomy.

In our case, the day before surgery, we embolized tumor feeding arteries to reduce intraoperative bleeding. Blood loss was $7,800 \mathrm{ml}$. It is reported that TAE for a giant tumor improves coagulopathy and increases the safety of surgery [4]. We think it is probable that we would not have been able to complete the operation due to hemorrhage if we had not performed preoperative TAE.

\section{Conclusion}

We performed a salvage right pneumonectomy for a huge pulmonary metastasis with PT and assisted circulation, and the patient lived more than 1 year. PT could be one of the choices for surgical incision in the case of a giant intrathoracic tumor. To our knowledge this is the first report of PT for a huge lung tumor.

\section{Conflict of interest}

The authors declare that they have no conflict of interest.

\section{References}

1. Incarbone M, Voulaz E, Alloisio M (2008) Surgical approach to giant thymoma: is the hemi-clamshell incision the best option?.Thorac Cardiovasc Surg 56: 241. [Crossref]

2. Bains MS, Ginsberg RJ, Jones II WG, McCormack PM, Rusch VW, et al. (1994) The clamshell incision: an improved approach to bilateral pulmonary and mediastinal tumor.Ann ThoracSurg 58: 30-33. [Crossref]

3. Ikeda S, Hatakenaka R, Matsubara Y, Kosaba S, Watabe S, et al. (1991) Plastron thoracotomy for a giant invasive thymic tumor. HaiganSyujyutsuSyugi 4:284-294.

4. Suzuki H, Nimura Y, Kamiyama J, Kondo S, Nagino M, et al. (1997) Preoperative transcatheter arterial embolization for giant cavernous hemangioma of the liver with consumption coagulopathy. Am J Gastroenterol 92: 688-691. [Crossref]

Copyright: (C2017 Fukai R. This is an open-access article distributed under the terms of the Creative Commons Attribution License, which permits unrestricted use, distribution, and reproduction in any medium, provided the original author and source are credited. 